\title{
Optimization IIR Filter Design Using Symbiotic Organism Search Algorithm
}

\author{
Cao $\mathrm{Han}^{1}$ and Yongquan Zhou ${ }^{2,3,{ }^{*}}$ \\ ${ }^{1}$ School of Computer, Electronics and Information, Guangxi University, Nanning 530004, China \\ ${ }^{2}$ College of Information Science and Engineering, Guangxi University for Nationalities, Nanning \\ 530006, China \\ ${ }^{3}$ Key Laboratory of Guangxi High Schools Complex System and Computational Intelligence, \\ Nanning 530006, China \\ *Corresponding author
}

Keywords: Symbiotic organism search algorithm, IIR filter, Global optimization.

\begin{abstract}
The possible multi-modal error surface is a challenge to the IIR filter design, which needs a flexible, easy to implement and stable global optimization algorithm to provide filter coefficient information. The Symbiotic Organism Search (SOS) algorithm is an effect global optimization algorithm which is proposed by Min-yuan Cheng in 2014. It simulates the symbiotic interaction between different populations in nature. In this paper, the IIR filter design in system recognition field based on the SOS algorithm will be described. Two IIR filters in different structures were used for experiment. The simulation results will be compared with some well-known algorithms that also provide solutions for IIR design. The comparative results show that: the design solution provided by the SOS algorithm is superior to Compared algorithms.
\end{abstract}

\section{Introduction}

Adaptive filters are with the powerful adaptability of modifying, reshaping or manipulating the frequency spectrum of a signal according to the desired response. Therefore, it is widely used in system model identification, noise cancellation, prediction spectrum and automatic equalization in the field of communication. Adaptive filters consist of two broad categories: recursive infinite impulse response (IIR) filter and non-recursive finite impulse response (FIR) filter. Generally, for the same number of coefficients, the former performs better than the latter [1]. More significantly, the lower order of the IIR filter makes it require only a small amount of computational overhead.

However, there is an important design challenge that the IIR filter can produce a multimodal error surface, which makes traditional filter methods misled by local optimum and fail to find global minimum error surface. An effect global optimization algorithm is needed to solve this difficulty. In recent ten years, meta-heuristic algorithms with natural evolutionary ideas have attracted more and more scholars' attention and tracking due to their speed, robustness and accuracy near the optimal solution. In particular, they can find the optimal values of multimodal functions by jumping out local minima. Therefore, various meta-heuristic algorithms have been employed to deal with the multimodal error plant in designing IIR filter. These effective algorithms are mainly: particle swarm algorithm (PSO) [2], artificial bee colony (ABC) algorithm [3], gravitational search algorithm (GSA) [4], bat algorithm (BA) [5] and cat algorithm (CA) [6].

Although the above algorithms provide a solution for IIR design, they are often unstable because of their own adjustable parameters. The Symbiotic Organism Search (SOS) algorithm [7] was first proposed by Min-Yuan Cheng in 2014 as a mate-heuristic optimization algorithm. The SOS algorithm is with simple structure, easily implement, no parameter, and higher stability. Once proposed, SOS algorithm is widely used in power systems, structural engineering, combinatorial optimization, scheduling problems, and so on [8]. In this paper, we try to employ the SOS algorithm for solving the filter design problem to expand the application of SOS.

The rest structure of the paper is as follows: Section 2 describes the SOS algorithm. The different structural IIR filters are presented in Section 3. Section 4 gives experimental results and analysis. 
What's more, SOS is compared with PSO, ABC, GSA, IF_ABC, CA and NBA. Finally, Section 5 provides some remarks and conclusions.

\section{Symbiotic Organisms Search (SOS) Algorithm}

According to the characteristics of symbiosis in nature, symbiotic relationship roughly falls into three types: mutualism, commensalism, and parasitism.

\section{Mutualism Phase}

Initially, an ecosystem of size $N$ is randomly formed. In the mutualism phase, for each $X_{i}, X_{j}$ (where $X_{j} \neq X_{i}$ ) is randomly selected from the ecosystem to establish relationship. $X_{i}$ reaps the benefits from $X_{j}$, and $X_{j}$ also benefits from $X_{i}$. Mutualism phase can be modeled as Eq.1 and Eq.2:

$$
\begin{aligned}
& X_{\text {inew }}=X_{i}+\operatorname{rand}(0,1) *\left(X_{\text {best }}-\text { Mutual_Vector } * B F_{1}\right) . \\
& X_{\text {jnew }}=X_{j}+\operatorname{rand}(0,1) *\left(X_{\text {best }}-\text { Mutual_Vector } * B F_{2}\right) .
\end{aligned}
$$

here $X_{\text {best }}$ symbolizes the most adaptive organism in the current iteration, which is evaluated by the fitness function. The two benefit coefficients, $B F_{1}$ and $B F_{2}$, reflect the degree of benefit of each other. Mutual_Vector denotes the process of getting close to each other, which is calculated as Eq. 3:

$$
\text { Mutual_Vector }=\frac{X_{i}+X_{j}}{2} \text {. }
$$

It is worth noting that $X_{i}$ and $X_{j}$ are replaced only if the updated fitness is better than their previous fitness.

\section{Commensalism Phase}

In this phase, for each individual $X_{i}, X_{j}$ (where $X_{j} \neq X_{i}$ ) is randomly selected from the ecosystem to establish relationship. Where, $X_{i}$ benefits from $X_{j}$. Therefore, only the $X_{i}$ individual location is updated:

$$
X_{\text {inew }}=X_{i}+\operatorname{rand}(-1,1) *\left(X_{\text {best }}-X_{j}\right) \text {. }
$$

The rand $(-1,1)$ function is a random number vector between -1 and 1 . Here $X_{i}$ is replaced only if the updated fitness is better than its previous fitness.

\section{Parasitism Phase}

The detailed implementation process of the parasitic phase is described as follows: First copying $X_{i}$ and then randomly modifying the values on several dimensions to produce Parasite_Vector .Calculate the fitness function values for Parasite_Vector and $X_{j}$. If the fitness value of Parasite_Vector is better, then Parasite_Vector replaces $X_{i}$; otherwise, Parasite_Vector is deleted.

\section{IIR Filter Design Problem}

In this section, we take the IIR filter in the field of system model identification as an example to show the working principle of the IIR filter. Fig. 1 shows the block diagram of the system model identification. $x(k)$ is actual input of the unknown plant and $d(k)$ is actual output of the unknown plant. $y(k)$ is the IIR filter's output. System model identification requires that the output $y(k)$ from the IIR is as close as possible to the actual output from the unknown plant. The relationship between the IIR filter input $x(k)$ and the output $y(k)$ is given below: 


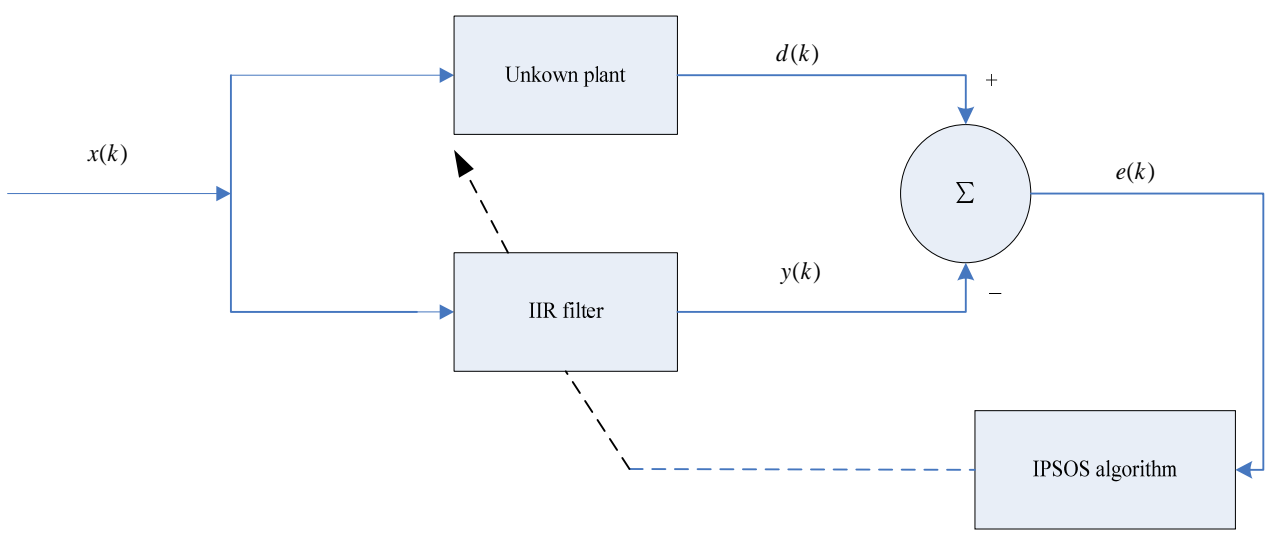

Figure 1. IIR filter in system identification

$$
y(k)+\sum_{i=1}^{M} a_{i} y(k-i)=\sum_{i=0}^{L} b_{i} x(k-i) .
$$

Where, $M$ is the order of the filter, a $(\mathrm{i}=1,2, \ldots, \mathrm{M})$ is the filter pole coefficient and $\mathrm{b}(i=0,1, \ldots$, $L)$ are the filter zero coefficient. The IIR filter transfer function is shown in the Eq.6:

$$
H(z)=\frac{B(z)}{A(z)}=\frac{\sum_{i=0}^{L} b_{i} z^{-i}}{1+\sum_{i=1}^{M} a_{i} z^{-i}} .
$$

Then, the IIR filter design is transformed into an optimization problem that is equal to minimize the difference between the unknown plant output and the filter output:

$$
J(w)=\frac{1}{N} \sum_{k=1}^{N}(d(k)-y(k))^{2} .
$$

where $w$ represents the coefficient vector to be adjusted, $w=\left(a, a_{2}, \mathrm{~K}, a_{L}, b_{0}, b_{1}, \mathrm{~K}, b_{M}\right) . \mathrm{N}$ represents the number of samples entered.

In this section, we consider two different orders IIR filters as simulation objects, and the transfer function $H_{s}(z)$ is for the unknown plant and the transfer function $H(z)$ is for IIR model.

(1) A plant with a Second-Order System and a Second-Order IIR $R_{1}$ Model is considered [1]:

$$
H_{s}(z)=\frac{1}{1-1.4 z^{-1}+0.49 z^{-2}} . \quad H(z)=\frac{b}{1+a_{1} z^{-1}+a_{2} z^{-2}} .
$$

(2) A plant with a superior-order System and a high-order $I I R_{2}$ Model is considered [1]:

$$
H_{s}(z)=\frac{1-0.4 z^{-2}-0.65 z^{-4}+0.26 z^{-6}}{1-0.77 z^{-2}-0.8498 z^{-4}+0.6486 z^{-6}} . \quad H(z)=\frac{b_{0}+b_{1} z^{-1}+b_{2} z^{-2}+b_{3} z^{-3}+b_{4} z^{-4}}{1+a_{1} z^{-1}+a_{2} z^{-2}+a_{3} z^{-3}+a_{4} z^{-4}} .
$$

\section{Comparison of Experiment Results}

In this section, we use the SOS algorithm to solve IIR filter design problem with two different structures mentioned in section 3. The obtained experimental results are compared with the results from GSA [4], ABC [3], IF_ABC [9], NBA [5], PSO [2] and CA [6].

\section{Simulation Experiment Platform and Parameter Setting}

For performance evaluation, results are taken using 64-bit Windows 7, Intel Core Xeon(R), CPU@ 3.5GHZ processor with 8 GB RAM and using MATLAB version R2012a. All algorithms settings are up to 500 iterations and run 30 times independently. All algorithms default to searching with a population size of 30.100 samples are considered $(\mathrm{N}=100)$ for the input $x(k)$ in four IIR filter design experiment. 


\section{Result Analysis}

Table 1. Performance comparison for IIR models design

\begin{tabular}{ccccccccc}
\hline \multirow{2}{*}{ IIR Model } & MSE & SOS & GSA & ABC & IF_ABC & NBA & PSO & CA \\
\hline \multirow{2}{*}{$I I R_{1}$} & Average & $4.8441 \mathrm{e}-31$ & 0.2089 & $3.8865 \mathrm{e}-03$ & $4.3967 \mathrm{e}-03$ & 0.0657 & 0.0592 & 0.0916 \\
& SD & $1.2791 \mathrm{e}-30$ & 0.0421 & $3.8859 \mathrm{e}-03$ & $6.5104 \mathrm{e}-03$ & 0.1067 & 0.09 & 0.104 \\
\hline \multirow{2}{*}{$I I R_{2}$} & Average & 0.00038803 & 0.0619 & 0.0493 & 0.0409 & 0.0278 & $5.5716 \mathrm{e}-03$ & 0.0223 \\
& SD & 0.00091394 & 0.0227 & 0.0157 & $9.3933 \mathrm{e}-03$ & 0.0269 & $5.7188 \mathrm{e}-03$ & 0.0284
\end{tabular}

In Experiment 1, since the system and the filter are both second-orders, there is no local minima problem. The average value and standard deviations of the best fitness function values in 30 running independently is used as the evaluation criterion. The simulation results are displayed in Table 1, it is surprised to discover that SOS found the smallest error plane (4.8441e-31), and the average fitness function value is much smaller than other algorithms. Fig. 2 and Fig. 3 give the convergence curve and ANOVA test for experiment 2, respectively. In Fig. 2, it is obvious that SOS converges fastest and reaches the minimum fitness value in the 20th iteration. After that, the curve is a smooth straight line and is infinitely close to the $\mathrm{X}$ axis without any fluctuations. The PSO and CA curves finally coincide to achieve the same accuracy, which is slightly less than that of SOS. From the ANOVA test, it is not difficult to find that the fitness function values of SOS are concentrated on a straight line, which reflects that the IIR design solution provided by SOS is very reliable. That also shows that the solutions provided by the NBA, CA, and PSO lack robustness.

Table 2. Results of the four IIR design problems

\begin{tabular}{ccccccccc}
\hline IIR Model & C & SOS & GSA & ABC & IF_ABC & NBA & PSO & CA \\
\hline \multirow{4}{*}{$I I R_{1}$} & $\mathrm{a}_{1}$ & -1.4 & -1.0887 & -1.4129 & -1.4013 & -1.4 & -1.4 & -1.3996 \\
& $\mathrm{a}_{2}$ & 0.49 & 0.1764 & 0.5012 & 0.4923 & 0.49 & 0.49 & 0.4896 \\
& $\mathrm{~b}$ & 1 & 0.8631 & 0.9933 & 1 & 1 & 1 & 1.0004 \\
\hline \multirow{6}{*}{$I^{*} R_{2}$} & $\mathrm{a}_{1}$ & -0.0857 & 0.238 & 0.6812 & 0.3965 & 0.0622 & 0.0547 & -0.4525 \\
& $\mathrm{a}_{2}$ & -0.3645 & -0.4241 & -0.4798 & -0.3630 & -0.4013 & -0.3831 & -0.2596 \\
& $\mathrm{a}_{3}$ & 0.0756 & -0.0673 & -0.7188 & -0.6718 & -0.0613 & -0.0542 & 0.3984 \\
& $\mathrm{a}_{4}$ & -0.5117 & -0.279 & -0.3461 & -0.3007 & -0.4356 & -0.4881 & -0.6259 \\
& $\mathrm{~b}_{0}$ & 0.9699 & 0.5667 & 0.8869 & 0.5794 & 0.9085 & 0.9796 & 0.9889 \\
& $\mathrm{~b}_{1}$ & -0.0928 & -0.0197 & 0.7542 & 0.2788 & 0.013 & 0.056 & -0.5987 \\
& $\mathrm{~b}_{2}$ & 0.0248 & -0.0779 & -0.2136 & 0.0081 & 0.0279 & -0.0077 & 0.1777 \\
& $\mathrm{~b}_{3}$ & 0.0404 & 0.2561 & -0.5206 & -0.5477 & -0.0044 & -0.01 & 0.3094 \\
& $\mathrm{~b}_{4}$ & -0.1935 & 0.4038 & -0.1501 & 0.1077 & -0.0201 & -0.1423 & -0.4494 \\
\hline
\end{tabular}




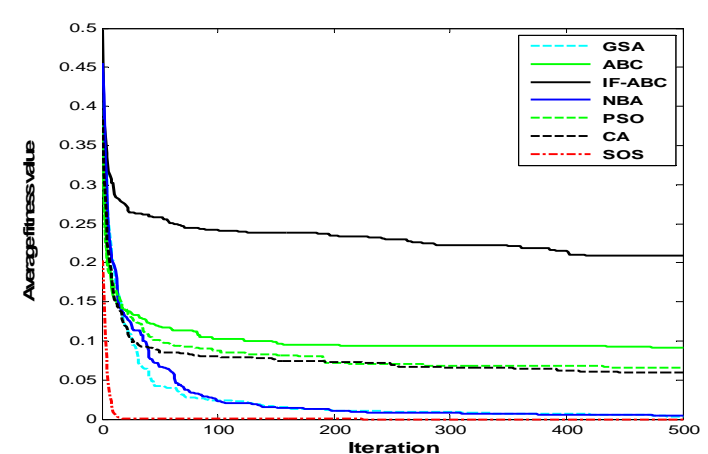

Figure 2. Convergence curves for $I I R_{1}$

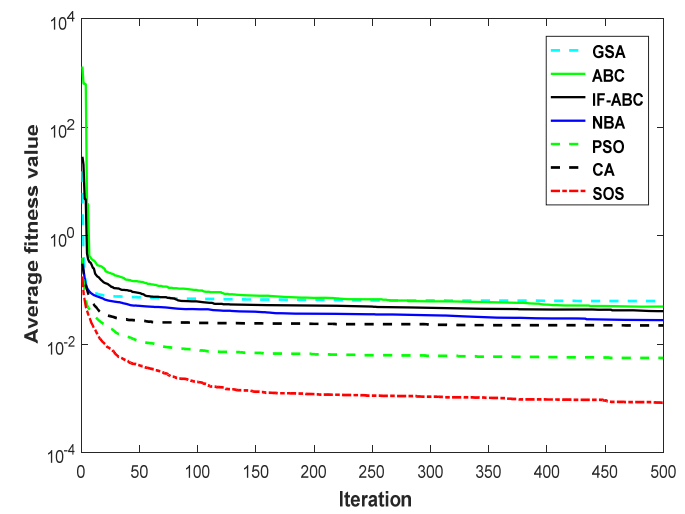

Figure 4.Convergence curves for $I I R_{2}$

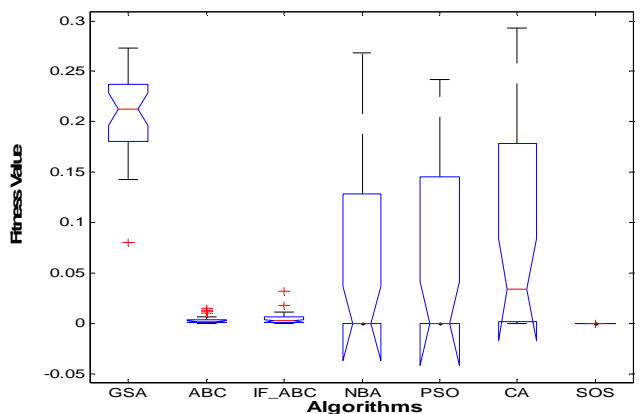

Figure 3. ANOVA tests for $I I R_{1}$

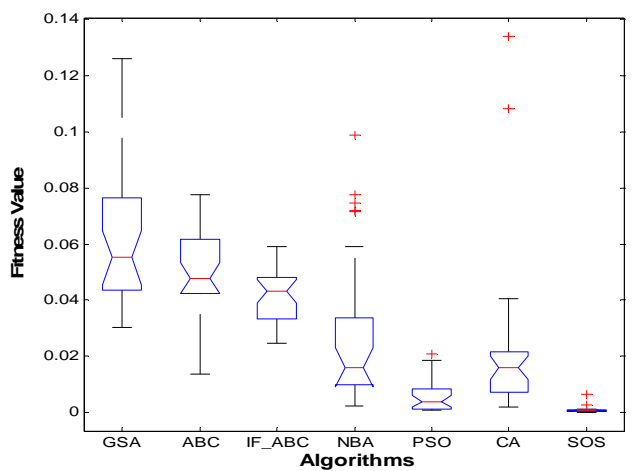

Figure 5. ANOVA tests for $I I R_{2}$

In Experiment 2, the search space for $I I R_{2}$ design is multimodal because the filter orders are smaller than the system. As in the previous experiment, Table 1 gives the results of the experiment. It shows that even if there is a multi-model error surface in the design of a high-order IIR, SOS still achieves the minimum average fitness value in all algorithms, and this result overwhelmingly defeats the other 6 algorithms. The standard deviation of SOS is also much smaller than other comparison algorithms. From the convergence curve of Fig. 4, it is easy to see that the curve performance of SOS is best in terms of convergence speed and accuracy. Also, Fig. 5 shows that the ANOVA test result of SOS is similar to PSO, but there are anomalies in PSO.

Table 2 lists the coefficient vectors corresponding to the minimum fitness function values of all algorithms. In general, Symbiotic Organism Search (SOS) algorithm can quickly find the minimum error plane in all comparison algorithms when solving the unimodal IIR filter design, and can provide stable filter parameter values. When solving the design of multi-model filter, SOS can effectively jump out of the local optima to achieve the most satisfactory error surface and the parameter fine-tuning ability is strong.

\section{Conclusions}

The Symbiotic Organism Search algorithm is used IIR filter design in the system identification in this paper. IIR filters of two different structures are employed for experiment, and their error surfaces contain both unimodal and multi-model. For simulation study, six well-known IIR filter design methods are compared. Moreover, the stability of the design solution by SOS algorithm was explored. Experiment result shows that Symbiotic Organism Search algorithm can effectively solve filter design problem and provide better solutions. So SOS algorithm is a effective method for global optimization. 


\section{Acknowledgments}

This work is supported by National Science Foundation of China under Grant No.61563008. Project of Guangxi University for Nationalities Science Foundation under Grant No. 2018 GXNSFAA138146.

\section{References}

[1] Kritele Loubna; Benhala Bachir; Zorkani Izeddine, Optimal digital IIR filter design using ant colony optimization. 2018 4th International Conference on Optimization and Applications (ICOA), IEEE, 2018.

[2]Aimi H, Suyama K. Multi-constrained IIR filter design using PSO. International Symposium on Electronics \& Smart Devices .IEEE, 2017.

[3] Karaboga N. A new design method based on artificial bee colony algorithm for digital IIR filters. Journal of the Franklin Institute, 2009, 346(4):328-348

[4] Gravitation search algorithm: Application to the optimal IIR filter design. Journal of King Saud University - Engineering Sciences, 2014, 26(1):69-81.

[5] X.B. Meng, X.Z. Gao, Y. Liu, et al., A novel bat algorithm with habitat selection and Doppler ect in echoes for optimization", Expert Systems with Applications, Vol.42, No.17, pp.6350\{6364, 2015.

[6] Sarangi A, Sarangi S K, Panigrahi S P. An approach to identification of unknown IIR systems using Crossover Cat Swarm Optimization. Perspectives in Science, 2016:S2213020916300817.

[7] Cheng M Y, Prayogo D. Symbiotic Organisms Search: A new metaheuristic optimization algorithm. Computers \& Structures, 2014, 139:98-112.

[8] Ezugwu A E, Prayogo D. Symbiotic Organisms Search Algorithm: theory, recent advances and applications. Expert Systems with Applications, 2019, 119:184-209

[9] B. Li, Y. Li and L.G. Gong. Protein secondary structure optimization using an improved artificial bee colony algorithm based on AB o_-lattice model. Engineering Applications of Artificial Intelligence, 2014, 27:70-79. 